\section{The role of the ventral pallidum in psychiatric disorders}

Largely garnered from studies in laboratory animals, the ventral pallidum (VP) is recognized as an integrator of sensory, emotional, and cognitive information with appropriate motoric responses. These functional complexities are reflected in behaviors associated with pain experiences, reward-motivated function, stress, social interaction and affiliation. With the increased resolution of modernday human brain imaging technology, preclinical studies are being substantiated, and the role of the VP in the human emotional repertoire and psychiatric disorders is being clarified.

Several clinical reports point to VP involvement in disorders of motivation. For example, single-photon emission computed tomography of Parkinson's Disease (PD) patients with pathological gambling show enhanced resting state activity (regional cerebral blood flow) in the VP of these individuals compared with non-gambling PD patients (Cilia et al, 2008).

Functional magnetic resonance imaging can assess rapid responses of the brain to 'unseen' reward cues or cues that are recognized outside our awareness. Presentation of unseen cues for natural and drug-related rewards (Childress et al, 2008), or monetary rewards (Pessiglione et al, 2007), results in rapid activation of the VP before conscious recognition. These rapid responses also predict the positive affect responses to the same stimuli when presented in a visible manner, suggesting that the affective/ motivational processes within and outside awareness are continuous and regulated by the VP (Childress et al, 2008).

Another behavior with high emotional valence is social affiliation, for example, pair bonding. Both the formation and the maintenance of pair bonding are associated with VP activation (assessed with structural magnetic resonance imaging and positron emission tomography) in non-human primates (Bales et al, 2007). It follows that a dysregulation of the VP and its limbic circuit may contribute to disorders of social bonding such as autism.

Positron emission tomography with receptor-selective radiotracers adds neurochemical assessments to studies on neuroanatomical substrates regulating emotional states. For example, increases in negative affect ratings by healthy human volunteers who are associated with sustained sadness (Zubieta et al, 2003) or sustained muscle pain (Zubieta et al, 2002) correlate with deactivation of $\mu$-opioid receptors in the VP. By inference, these findings indicate that positive affect is regulated by activated $\mu$-opioid receptors in the VP.

The imaging literature converges to indicate that VP transmission is involved in the emotional overlays of motivated behaviors. This interpretation is substantiated by preclinical studies on $\mu$-opioid receptors in the VP, wherein these receptors are capable of inducing a profound and enduring plasticity (Mickiewicz et al, 2009). Future laboratory and clinical studies will aid in fully appreciating the role of the VP in emotional processing and motivated behaviors, and the psychiatric consequence of its dysregulation.

T Celeste Napier ${ }^{1,2}$ and

Amanda L Mickiewicz ${ }^{1,3}$

${ }^{1}$ Center for Compulsive Behavior and Addiction, Rush University Medical Center, Chicago, IL, USA;

${ }^{2}$ Department of Pharmacology, Rush University Medical Center, Chicago, IL, USA;

${ }^{3}$ Department of Neurological Sciences, Rush University Medical Center, Chicago, IL USA

E-mail: celeste_napier@rush.edu

\section{DISCLOSURE}

The authors declare no conflict of interest.

Bales KL, Mason WA, Catana C, Cherry SR, Mendoza SP (2007). Neural correlates of pairbonding in a monogamous primate. Brain Res 1184: 245-253.
Childress AR, Ehrman RN, Wang Z, Li Y, Sciortino N, Hakun $J$ et al (2008). Prelude to passion: limbic activation by 'unseen' drug and sexual cues. PLOS One 3: e1506.

Cilia R, Siri C, Marotta G, Isaias IU, De GD, Canesi M et al (2008). Functional abnormalities underlying pathological gambling in Parkinson disease. Arch Neurol 65: 1604-1611.

Mickiewicz AL, Dallimore JE, Napier TC (2009). The ventral pallidum is critically involved in the development and expression of morphine-induced sensitization. Neuropsychopharmacology 34: 874-886.

Pessiglione M, Schmidt L, Draganski B, Kalisch R, Lau H, Dolan RJ et al (2007). How the brain translates money into force: a neuroimaging study of subliminal motivation. Science $\mathbf{3 1 6}$ : 904-906.

Zubieta JK, Ketter TA, Bueller JA, Xu Y, Kilbourn MR, Young EA et al (2003). Regulation of human affective responses by anterior cingulate and limbic mu-opioid neurotransmission. Arch Gen Psychiatry 60: 1145-1153.

Zubieta JK, Smith YR, Bueller JA, Xu Y, Kilbourn MR, Jewett DM et al (2002). mu-opioid receptormediated antinociceptive responses differ in men and women. J Neurosci 22: 5100-5107.

Neuropsychopharmacology Reviews (2010) 35, 337; doi: 10.1038/npp.2009.1 13

\section{Exploring the molecular basis of addiction: drug-induced neuroadaptations}

Abuse of a number of psychoactive substances can eventually control an individual's behavior by producing dependence and/or addiction. Recent surveys estimate that there are about 200 million users of illegal drugs worldwide, which represent $3.4 \%$ of the world population. An ever-increasing number of neuroscientists are searching for clues regarding the molecular determinants of addictive behavior. The low-hanging fruit would be to study dopamine receptors and transporters in the nucleus accumbens (NAc); however, scientists are now exploring mechanisms far beyond dopaminergic targets.

For example, some scientists have chosen to target molecular mechanisms within the hippocampus because of its role in encoding and retrieving 
information in the central nervous system. In addition, this brain region sends and receives projections from the mesolimbic dopamine system so often implicated in addiction (Bannerman et al, 2004). Further, the hippocampus has been directly implicated in addiction behavior (Vorel et al, 2001), likely because of the fact that there is increasing evidence suggesting that drug addiction represents a conditioning phenomenon that is largely dependent on associations between drug effects and environmental cues (Berke and Hyman, 2000).

Coordinated hippocampal/accumbal regulation is likely at the heart of the protective addiction phenotype produced by environmental enrichment. We find that rats reared in an enriched condition (large cages with cohorts and novel objects) self-administer cocaine or amphetamine less readily than rats in the isolated control group (Green et al, 2009). This protective phenotype is likely mediated by a coordinated decrease in cAMP response element binding protein (CREB) activity in the accumbens coupled with an increase in the hippocampus. Further, CREB has been linked to neuronal excitability (Dong et al, 2006), suggesting that some yet to be identified CREB target gene may produce this protective phenotype by decreasing excitability in the accumbens and increasing excitability in the hippocampus.

Addiction-related molecular targets in the hippocampus are also being interrogated using proteomics approaches. It is known that exposure to drugs of abuse alters the expression of certain synaptic proteins. Indeed, a recent study uses this state-of-the-art technology to examine the effects of morphine administration on the protein expression profile at hippocampal synapses (Moron et al, 2007). This study finds that repeated morphine administration alters the synaptic distribution of endocytic proteins. This finding has functional implications, as receptor trafficking largely depends on endocytosis, and therefore morphine may alter receptor localization by affecting synaptic redistribution of the endocytic machinery. The idea that endocytic proteins, such as clathrin, may be involved in morphine-induced changes at hippocampal synapses is quite innovative and opens up a new direction for the study of the mechanisms underlying morphine-induced neuroadaptations. In addition, these findings suggest that the study of hippocampal neuroadaptations induced by repeated morphine treatment has great potential to reveal the mechanisms contributing to the development of opiate addiction.

Addiction is a complex polygenic psychiatric condition involving many brain regions, proteins and physiological effects, not to mention varied etiologies. Understanding the molecular mechanisms underlying addictive behavior will someday allow for therapeutic intervention that will be both efficacious and safe.

Jose A Morón ${ }^{1}$ and Thomas A Green ${ }^{1}$

${ }^{1}$ Department of Pharmacology \& Toxicology, Center for Addiction Research, The University of Texas Medical Branch, Galveston, TX, USA

E-mail: jomoronc@utmb.edu or thgreen@utmb.edu

\section{DISCLOSURE}

The authors declare that except for income received from their primary employer, no financial support or compensation has been received from any individual or corporate entity over the past 3 years for research or professional services, and there are no financial holdings that could be perceived as constituting a potential conflict of interest.

Bannerman DM, Rawlins JN, McHugh SB, Deacon RM, Yee BK, Bast $T$ et al (2004). Regional dissociations within the hippocampus-memory and anxiety. Neurosci Biobehav Rev 28: 273-283.

Berke JD, Hyman SE (2000). Addiction, dopamine, and the molecular mechanisms of memory. Neuron 25: 515-532.

Dong Y, Green T, Saal D, Marie H, Neve R, Nestler EJ et al (2006). CREB modulates excitability of nucleus accumbens neurons. Nat Neurosci 9: 475-477.

Green TA, Alibhai IN, Roybal CN, Winstanley CA, Theobald DE, Birnbaum SG et al (2009). Environmental enrichment produces a consistent behavioral phenotype indicative of decreased cAMP response element transcriptional activity in the nucleus accumbens. Biol Psychiatry (in press).

Moron JA, Abul-Husn NS, Rozenfeld R, Dolios G, Wang R, Devi LA (2007). Morphine administration alters the profile of hippocampal postsynaptic density-associated proteins: a proteomics study focusing on endocytic proteins. Mol Cell Proteomics 6: 29-42.
Vorel SR, Liu X, Hayes RJ, Spector JA, Gardner EL (2001). Relapse to cocaine-seeking after hippocampal theta burst stimulation. Science 292 1175-1178.

Neuropsychopharmacology Reviews (2010) 35 , 337-338; doi: 10.1038/npp.2009.106

\section{The emerging role of integrins in neuropsychiatric disorders}

Disrupted physical and functional associations between neurons are thought to govern the abnormal patterns of neural signaling that underlie neuropsychiatric disorders. As membrane signaling proteins that bridge extracellular interactions and intracellular signaling, integrins have been well studied for their role in cell migration, cell adhesion, and metastasis, but have been relatively underexplored for their contribution to mental illness and pharmacotherapy.

Integrins are obligate $\alpha$ - and $\beta$ heterodimers that display molecular heterogeneity, as well as developmental and regional regulation (Denda and Reichardt, 2007). Early in development, integrin signaling supports developmental events such as neuronal migration and synaptic differentiation. Integrins also contribute to the plasticity of mature synapses. In turn, both integrin expression and signaling are sensitive to drug and pathophysiological manipulation of synapses. For example, integrin subunit expression is differentially altered by acute and chronic cocaine administration (Wiggins et al, 2009). Such alterations are likely to be functionally important, as integrin expression can influence neuronal recovery after injury, a capacity that depends on the interaction of integrins with growth factor receptors and cytokines.

Several integrins have been associated with neuropsychiatric disorders, raising the possibility that they represent important new targets for 